\title{
ESTUDIOS DE ASPECTOS RELEVANTES DEL ENVEJECIMIENTO DE UNA POBLACIÓN DE ADULTOS MAYORES QUE PARTICIPAN EN UN PROGRAMA EDUCATIVO Y EL IMPACTO DE ESTE EN SU CALIDAD DE VIDA. OCTUBRE 2000.
}

Wender Florencio Condori Chipana'

\author{
RESUMEN
}

El control de la natalidad y el aumento en la esperanza de vida están produciendo un importante aumento de la población de la tercera edad, tendencia que se acentuará en las próximas décadas.

El envejecimiento es un proceso dinámico, progresivo e irreversible, que trae consigo la declinación de habilidades y capacidades que se debe más a la falta de entrenamiento y al desinterés que al proceso mismo del desgaste biológico normal, por eso la necesidad de redefinir la vejez como una etapa de la vida llena de posibilidades.

El envejecimiento demográfico implica replantearse politicas sociales, económicas, de salud y educativas, por lo que este proyecto es considerado de vital importancia, ya que considera que la EDUCACIÓN permite la permanente integración y participación de las personas y es prioridad en cualquier programa de mejoramiento de la calidad de vida de las personas, ya que entrega herramientas necesarias para que las personas sean agentes de su propio desarrollo y bienestar, posibilitando que las personas mantengan su autonomía física y mental hasta edades avanzadas, a la vez logren un "ENVEJECIMIENTO CON ÉXITO".

\section{A B STRACT}

Control of natality and the increment in life expectations are producing an important increase in the thirf age population, tendency which will be accentuated in the next decades. Gelting older is a dynamic, progresive and irreversi ble process which carries the declinnig of skills and capacities because of the lack of training and theh inditherence than to the normal biological waste process, becuse of this the necessity of redefine the old age as a stage of life, full of possibilities.

The demographic aging implies to reconsider social, economical, health and educational policies, that is why this sesearch paper is of vital imortance.

We cousider that education allows permanent people 's integration and participation and it is priority in any program oriented to improve people 's life quality because it gives the necessary tools to make people be agents of their ocon development and well-bening, facilitatuig people to keep their plupical and mental autonomy even in advanced ages, to get their "Successfull Aging" at the same time.

1. Enfermero. 


\section{INTRODUCCIÓN}

El descenso de la fecundidad, de la mortalidad y la migración acompañado del incremento de la esperanza de vida ha aumentado en forma notable la población a partir de 1950. Las proyecciones actuales indican que el año 2025 más de la mitad de los paises latinoamericanos habrán de experimentar un envejecimiento sustancial de la población en su estructura por edades. La magnitud y la rapidez de este cambio demográfico impone grandes retos a las instituciones económicas y de salud a los países de la región.

Entre los aspectos en que su efecto se hará evidente de manera especialmente acentuada están los sistemas de pensión y jubilación, la composición de la fuerza laboral, las estructuras familiares, las transferencias intergeneracionales dentro de las familias, las condiciones sanitarias y las condiciones de salud de los adultos mayores.

El envejecimiento tendrá características diferentes de acuerdo a las condiciones de las distintas poblaciones en los países del continente. Por ejemplo: la población de Chile, Costa Rica, Uruguay y Argentina presentan un envejecimiento más temprano que Brasil, México y Perú, pero el proceso de envejecimiento en estos últimos ocurrirá en un período más reducido.

Al proceso de envejecimiento se asocia un deterioro gradual de las condiciones de salud física y mental de las personas, por lo que es previsible una reducción de los años de esperanza de vida activa y saludable.

El Perú vive, al igual que otros países del mundo, un acelerado proceso de envejecimiento demográfico. En la actualidad cuenta con una población de 24818942 de habitantes, siendo la población adulta mayor 1737326 personas, constituyendo el $7 \%$ del total, en donde el $48 \%$ son varones y el $52 \%$ son mujeres, de los cuales el $70 \%$ habitan en la zona urbana y el $30 \%$ en la zona rural.

Es más, para el año 2020 la población adulta mayor ascenderá a 3704114 correspondiendo el $11 \%$ de la población total.

En Tacna el crecimiento poblacional esta en un incremento de 218353 el $4,2 \%$ a 396174 el 5,5\%, para el año 2000, y la esperanza de vida aumentará a 76 años para el 2015 y referente a la prestación de servicios de salud el 53,2\% no recibe atención, debido a que nos es asegurada y sus ingresos no cubren los gastos de prevención y tratamiento de enfermedades y/o accidentes, contribuyendo a una discriminación arbitraria familiar, laboral y social, a lo que se asocia la falta de políticas de apoyo y de recursos especializados.

La revolución demográfica implica replantear políticas sociales, sanitarias, económicas y educacionales, por lo que este proyecto es de vital importancia, ya que considera, que la educación es la única herramienta de cambio de estilo de vida y que permite el mejoramiento de la calidad de vida de los adultos mayores, posibilitando el mantenimiento de su autonomía física, mental y social hasta edades avanzadas.

\section{2.- OBJETIVO GENERAL}

Determinar algunos aspectos más relevantes del envejecimiento de una población de adultos mayores, que participan en un programa educativo y el impacto de este en la calidad de vida.

\section{OBJETIVOS ESPECÍFICOS}

a.- Indagar sobre los factores sociodemográficos: edad, sexo, estado civil, grado de instrucción, ocupación y participación social de la población de adultos mayores, que participan en el programa educativo.

b.- Indagar sobre los factores afectivos: satisfacción con la vida, percepción de bienestar de la población de adultos mayores, que participan en el programa educativo.

c.- Evaluar cualitativamente, si la educación impartida ha contribuido en mejorar las acciones de su propio autocuidado de la población de adultos mayores, que participan en el programa educativo.

d.- Evaluar costo / beneficio del programa educativo en la calidad de vida, de la población de adultos mayores que participan en el programa educativo.

\section{3.- MATERIAL Y MÉTODOS}

Este trabajo de investigación fue efectuado, por el propio gestor del proyecto, elaborándose 
previamente un instrumento en cuyo contenido se han establecido datos relevantes como:

a.- Factores sociodemográficos: edad, sexo, estado civil, grado de instrucción, ocupación, y la participación social del adulto mayor.

b.- Factores afectivos: satisfacción con la vida, su percepción de bienestar del adulto mayor.

c.- Factores relacionados con la salud: el autocuidado del adulto mayor

El instrumento se aplicó al $88 \%$ de estudiantes adultos mayores asistentes a clases un día cualquiera, durante la finalización del segundo semestre.

\section{RESULTADOS}

El presente estudio intenta contribuir un mayor conocimiento sobre los factores que influyen en la calidad de vida del adulto mayor:

\section{A.- FACTORES SOCIODEMOGRÁFICOS.}

a.1. El $53 \%$ son adultos mayores cuyas edades oscilan entre 60 y 80 años de edad.

a.2. El $74 \%$ de los estudiantes adultos mayores son de sexo femenino.

a.3. El $73 \%$ son casados.

a.4. El $57 \%$ tienen superior incompleta.

a.5. El $69 \%$ son jubilados y cesantes como ocupación actual.

a.6. El $47 \%$ son jubilados del sector Educación.

a.7. El $100 \%$ esta predispuesto en participar en organizaciones de adultos mayores.

a.8. El $57 \%$ participaría en forma remunerada.

a.9. El $99 \%$ ha mejorado sus relaciones interpersonales con su entorno familiar y social.

\section{B.- FACTORES AFECTIVOS DE SATISFACCIÓN CON LA VIDA Y SU PERCEPCION DE BIENESTAR.}

b.1. El $50 \%$ ingreso al programa, por mejorar e incrementar su desarrollo personal. b.2. El $100 \%$ ha presentado cambios en su vida desde el momento que asistió al programa.

b.3. El $70 \%$ ha mejorado su autoestima.

b.4. El $100 \%$ ha mejorado su estado de ánimo.

b.5. El $99 \%$ ha cambiado su imagen sobre el envejecimiento, vejez y viejo.

\section{FACTORES RELACIONADOS CON LA SALUD AUTOCUIDADO.}

El $94 \%$ esta más seguro y actualizado, sobre las acciones del autocuidado de su salud.

\section{CONCLUSIONES}

1.- Que, las condiciones sociodemográficas, afectivas y educacionales son factores determinantes en la calidad de vida de nuestro envejecimiento.

2.- Que, la revolución demográfica se refleja en la mayor proporción de mujeres al de los hombres, lo indica la feminización del envejecimiento, siendo proclives a la discriminación: económicamente, en la asistencia sanitaria, en la protección socia, por genero y edad.

3.- Que, el factor psicosocial Afectivo, juega un papel relevante en la calidad de vida, el solo hecho de participar en un programa de indole universitario ha incrementado su autoestima y el estado de animo del aduito mayor.

4.- Que, las acciones de autocuidado dirigidas a la Promoción y Prevención de la salud mejoran las condiciones de vida sobre todo en la funcionalidad física, psiquica y social.

5.- Que, la eficiencia y la efectividad del programa educativo se ha percibido en el cambio de estilo de vida de los adultos mayores, desterrando los estereotipos negativos considerados como: decrépitos, incompetentes y frágiles.

6.- Que, la participación es un factor clave para el desarrollo social de una comunidad. 


\section{REFERENCIAS BIBLIOGRÁFICAS}

Belsky, Janet K.; Psicología del Envejecimiento.

Bermejo Garcia, L. Atención Sociosanitaria para personas mayores dependientes. Consulting Dubal, 1999.

Gerontología Básica. Lecturas Complementarias. Dirección de Educación a Distancia TELEDUC. Pontificia Universidad Católica de Chile. Segunda Edición. 1993.

Marin, Pedro P.: Tiempo Nuevo para el Adulto Mayor. Pontificia Universidad Católica de Chile. Segunda Edición. 1993.

Medina, Jhon J.: EI Reloj de la Edad. CRITICA (Grijalbo Mondadori, S. A ). Barcelona. 1997.

Ministerio de Salud de Chile. Cuidado de la Salud del Adulto Mayor. Programa de Salud del Adulto. 1996.
Steven N., Austad.: Porqué Envejecemos. Paidós |bérica. Barcelona. 1998.

Yaguas,Javier $\mathrm{J}$ y otros.: Intervención Psicosocial en Gerontologia.Cáritas.

Lira Andrade, Javier. Tacna, Realidad Social de la Tercera Edad. 1998.

Revistas:

SESENTA Y MAS . Publicación del Ministerio de Trabajo y Asuntos Sociaies-Secretaria General de Asuntos Sociales. IMSERSO- No 189.190.191.

UNIVERSIDAD POPULAR, Publicación del Centro de Capacitación de Adultos. "Alfonso López P", Cali N³ -1997.

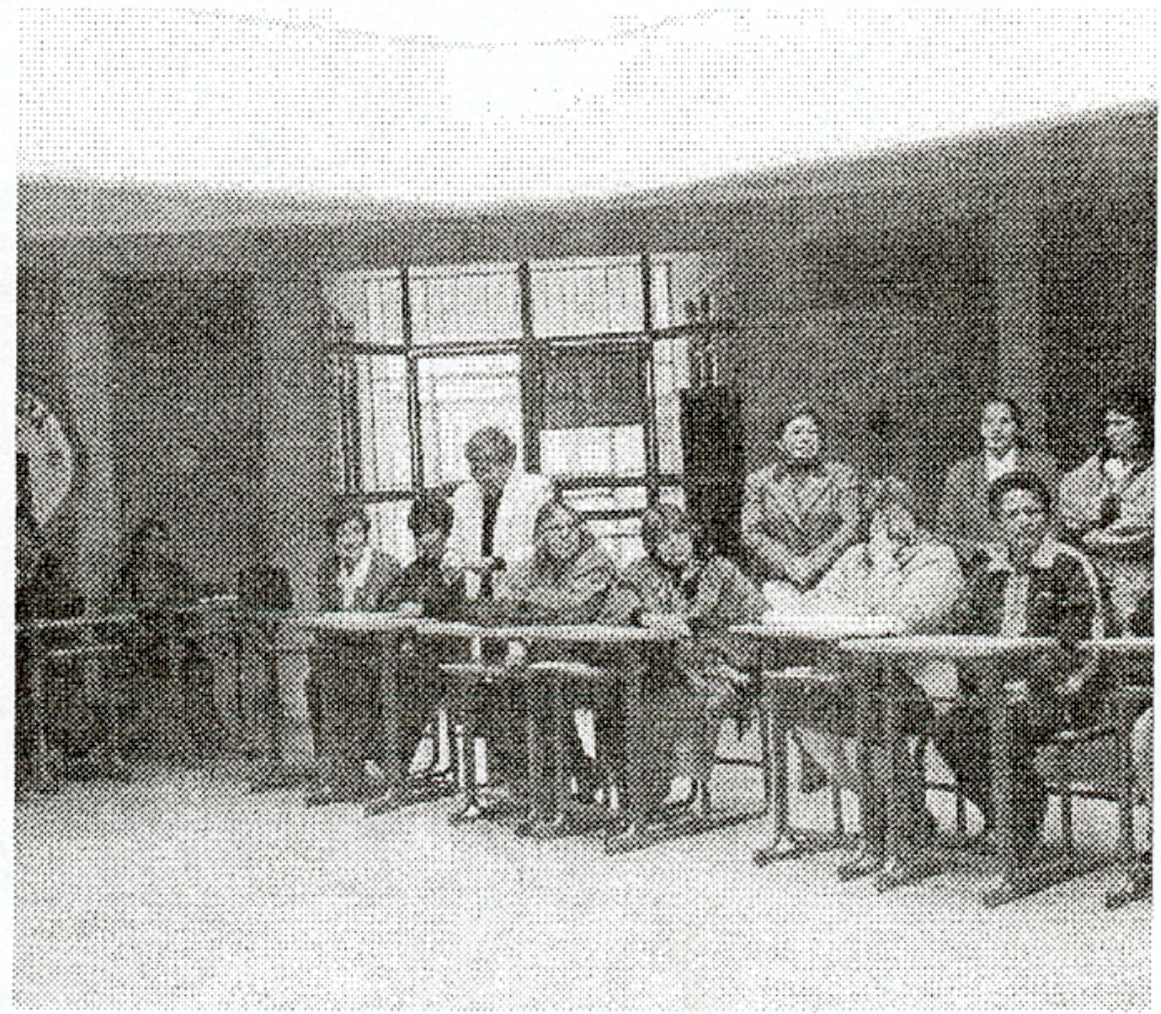

Foto 1. Adultos mayores participando en un programa educativo. 